\title{
The Algebra and Geometry of Curve and Surface Inversion
}

Thomas W. Sederberg

tom@cs.byu.edu

Eng-Wee Chionh

Kent Ridge

Follow this and additional works at: https://scholarsarchive.byu.edu/facpub

Part of the Computer Sciences Commons

\section{Original Publication Citation}

T.W. Sederberg and E.W. Chionh, "The algebra and geometry of curve and surface inversion," Geometric Modeling and Processing, 22.

\section{BYU ScholarsArchive Citation}

Sederberg, Thomas W.; Chionh, Eng-Wee; and Ridge, Kent, "The Algebra and Geometry of Curve and Surface Inversion" (2002). Faculty Publications. 1075.

https://scholarsarchive.byu.edu/facpub/1075 


\section{The Algebra and Geometry of Curve and Surface Inversion}

\author{
Thomas W. Sederberg \\ Department of Computer Science \\ Brigham Young University \\ Provo, Utah 84602 \\ tom@cs.byu.edu
}

\author{
Eng-Wee Chionh \\ School of Computing \\ National University of Singapore \\ Kent Ridge, Singapore 117543 \\ chionhew@ comp.nus.edu.sg
}

\begin{abstract}
An inversion equation takes the Cartesian coordinates of a point on a parametric curve or surface and returns the parameter value(s) of that point. A 2-D curve inversion equation has the form $t=f(x, y) / g(x, y)$. This paper shows that practical insight into inversion can be obtained by studying the geometry of the implicit curves $f(x, y)=0$ and $g(x, y)=0$. For example, the relationship between the singular locus of the parametric curve and the lowest possible degree of an inversion equation can be understood in this way. Also, insight is given into what parameter value will be returned if an inversion equation is fed the Cartesian coordinates of a point that does not lie on the curve.

The standard method of devising curve and surface inversion equations is as a by-product of the implicitization process. This paper presents a new method for finding inversion equations, which allows us to create new inversion equations that have attractive properties. For example, we can create an inversion equation that, to first order approximation, will return the parameter value of the nearest point on the curve if given a point that does not lie precisely on a parametric curve.
\end{abstract}

\section{Introduction}

Given a point $(x, y)$ on the degree $n$ rational plane curve

$$
\mathbf{P}(t)=\left(\frac{a(t)}{c(t)}, \frac{b(t)}{c(t)}\right)=\left(\frac{\sum_{i=0}^{n} a_{i} t^{i}}{\sum_{i=0}^{n} c_{i} t^{i}}, \frac{\sum_{i=0}^{n} b_{i} t^{i}}{\sum_{i=0}^{n} c_{i} t^{i}}\right)
$$

where $\operatorname{gcd}(a(t), b(t), c(t))=1$, the process of finding the corresponding parameter $t$ such that $\mathbf{P}(t)=(x, y)$ is known as inversion $[4,8]$. It is well-known that for a proper parametrization (that is, the correspondence $t \rightarrow(x, y)$ is one-to-one for all except finitely many $t$ ), polynomials $f(x, y)$ and $g(x, y)$ exist such that

$$
t=\frac{f(x, y)}{g(x, y)}
$$

for all but finitely many $(x, y)$. A major theme of this paper is that valuable insight emerges when studying the implicit curves $f(x, y)=0$ and $g(x, y)=0$.

One way of finding an inversion formula is to take the negative ratio of adjacent first minors of an implicitization matrix. For example, consider the unit circle about the origin

$$
\mathbf{P}(t)=\left(\frac{t^{2}-1}{t^{2}+1}, \frac{2 t}{t^{2}+1}\right) .
$$

The Sylvester implicitization matrix is

$$
\left[\begin{array}{cccc}
x-1 & 0 & x+1 & 0 \\
0 & x-1 & 0 & x+1 \\
y & -2 & y & 0 \\
0 & y & -2 & y
\end{array}\right]
$$

The matrix $M$ whose $(i, j)$ entry $M_{i, j}$ is the $(i, j)$ first minor of the Sylvester matrix is

$$
2\left[\begin{array}{cccc}
-y^{2}+2 x+2 & -y(x+1) & y^{2} & (x-1) y \\
y(x+1) & -y^{2} & -(x-1) y & y^{2}+2 x-2 \\
y(x+1) & \left(x^{2}-1\right) & -(x-1) y & -(x-1)^{2} \\
-(x+1)^{2} & y(x+1) & \left(x^{2}-1\right) & -(x-1) y
\end{array}\right] .
$$

Each of the twelve negative ratios of adjacent minors $-M_{i, j} / M_{i, j+1} ; i=1,2,3,4 ; j=1,2,3$; gives an inversion formula for the circle. Here only four ratios are distinct:

$$
\frac{-y^{2}+2 x+2}{y(x+1)}, \frac{(x+1)}{y}, \frac{y}{1-x}, \frac{(x-1) y}{y^{2}+2 x-2} .
$$

The implicitization Bezout matrix behaves especially nice [3]. For its first minors, we have $M_{i, j}=M_{k, l}$ when $i+j=k+l$ so we might as well write $M_{i, j}=M_{i+j}$. The Bezout matrix for the unit circle is

$$
\left[\begin{array}{cc}
-2 x+2 & -2 y \\
-2 y & 2 x+2
\end{array}\right]
$$


The corresponding first minor matrix $M$ is

$$
\left[\begin{array}{cc}
2 x+2 & -2 y \\
-2 y & -2 x+2
\end{array}\right] .
$$

The ratios $-M_{p} / M_{p+1}$ where $p=2,3$ give the inversion formulas

$$
t=\frac{x+1}{y}, \quad t=\frac{y}{1-x} .
$$

Note that the line $x+1=0$ intersects the circle twice at $\mathbf{P}(0)=(-1,0)$, the line $y=0$ intersects the circle at $\mathbf{P}(0)$ and $\mathbf{P}(\infty)=(1,0)$, and the line $1-x=0$ intersects the circle twice at $\mathbf{P}(\infty)$. The three lines and their intersection with the circle are shown in Figures 1.

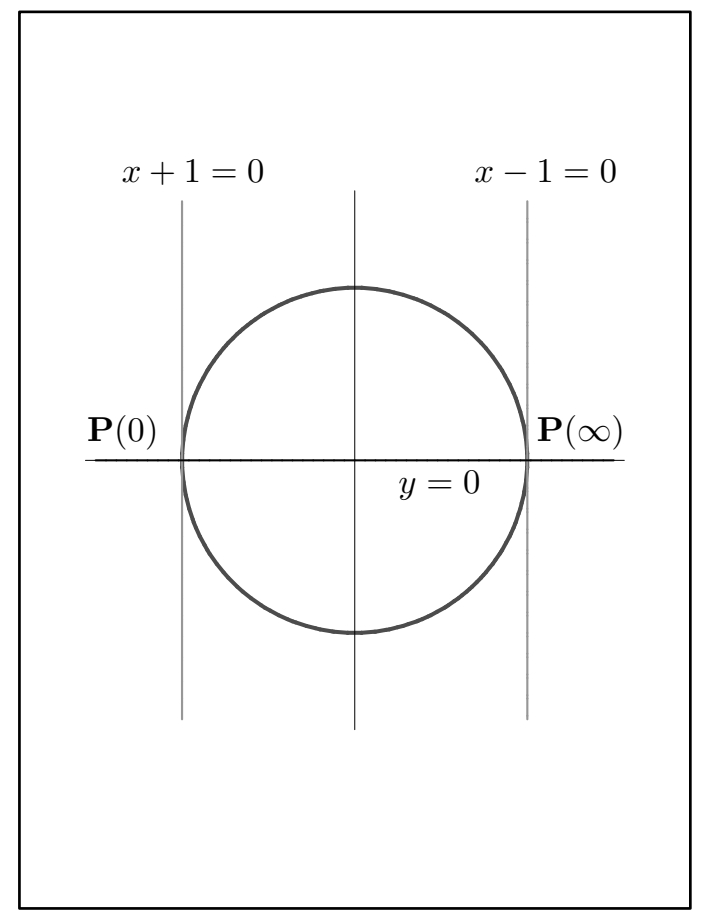

Figure 1. The Bezout matrix gives The inversion lines $x+1=y=0$ and $y=1-x=0$ for the unit circle.

The main objective of this paper is to explore alternatives in finding inversion formulas that possess some desirable qualities. In particular, we look for lowest degree inversion, inversion of fat curves, and flatter inversion curves. To this end the paper has the following sections. Section 2 examines the algebra and geometry of inversion. Section 3 seeks the lowest degree inversion for plane curves. Section 4 finds the nearest parameter value for a point near a plane curve. Section 5 studies the task of making inversion curves flatter. Section 6 inverts space curves and Section 7 inverts surfaces. Section 8 concludes the paper with a summary and some open questions.

\section{The Algebra and Geometry of Inversion}

In order to include points at infinity, we enlarge the affine parameter line $t$ to the projective parameter line $(t: s)$ and the affine plane $(x, y)$ to the projective plane $(x: y: w)$. In this embedding we identify the affine parameter $t / s$ with the projective parameter $(t: s), s \neq 0$ and the projective point $(1: 0)$ is denoted as $t=\infty$. Similarly, the affine point $(x / w, y / w)$ is identified with the projective point $(x$ : $y: w), w \neq 0$. In this projective setting, the parametric curve becomes

$$
\mathbf{P}(t, s)=\left(\frac{a(t, s)}{c(t, s)}, \frac{b(t, s)}{c(t, s)}\right)
$$

or

$$
\mathbf{P}(t, s)=(a(t, s): b(t, s): c(t, s))
$$

where $a, b, c$ now denote homogeneous polynomials of the same degree $n$; and the inversion formula (2) becomes

$$
\frac{t}{s}=\frac{f(x, y, w)}{g(x, y, w)}
$$

where $f(x, y, w), g(x, y, w)$ are now homogeneous polynomials of the same degree $m$.

Example. The unit circle centered at the origin given projectively is

$$
\mathbf{P}(t, s)=\left(t^{2}-s^{2}: 2 s t: t^{2}+s^{2}\right)
$$

or

$$
\mathbf{P}(t, s)=\left(\frac{t^{2}-s^{2}}{t^{2}+s^{2}}, \frac{2 s t}{t^{2}+s^{2}}\right) .
$$

The inversion formulas from the Sylvester minors become

$$
\begin{gathered}
t=\frac{-y^{2}+2 w x+2 w^{2}}{y(x+w)}, \quad t=\frac{(x+w)}{y}, \\
t=\frac{y}{w-x}, \quad t=\frac{(x-w) y}{y^{2}+2 w x-2 w^{2}}
\end{gathered}
$$

The following theorem characterizes the polynomials $f$ and $g$ in the inversion formula.

Theorem 1 If $t=f(x, y) / g(x, y)$, then the curves $f(x, y)=0$ and $g(x, y)=0$ intersect $\mathbf{P}$ at the same points with the same multiplicities except $f=0$ intersects $\mathbf{P}(0)$ one more time than $g=0$ does but $f=0$ intersects $\mathbf{P}(\infty)$ one less time than $g=0$ does.

\section{Proof:}

Clearly, Formula (12) inverts $\mathbf{P}$ if and only if

$$
\frac{f(a(t, s), b(t, s), c(t, s))}{g(a(t, s), b(t, s), c(t, s))} \equiv \frac{t}{s} .
$$


Geometrically, the solutions of $f(a(t, s), b(t, s), c(t, s))=$ 0 and $g(a(t, s), b(t, s), c(t, s))=0$ give respectively the intersections of the curves $f(x, y, w)=0$ with $\mathbf{P}(t, s)$ and $g(x, y, w)=0$ with $\mathbf{P}(t, s)$. By Bezout's theorem, there are $m n$ intersections in each case if the number of intersections is finite. Thus we must have

$$
f(a(t, s), b(t, s), c(t, s)) \equiv t^{\alpha} s^{\beta} \prod_{i=1}^{m n-\alpha-\beta}\left(\sigma_{i} t-\tau_{i} s\right),
$$

and

$g(a(t, s), b(t, s), c(t, s)) \equiv t^{\alpha-1} s^{\beta+1} \prod_{i=1}^{m n-\alpha-\beta}\left(\sigma_{i} t-\tau_{i} s\right)$.

The above equations prove the theorem.

The above proof also reveals how the three curves $f(x, y)=0, g(x, y)=0$, and $\mathbf{P}(t)$ intersect.

Theorem 2 The common intersections of the three curves $f(x, y)=0, g(x, y)=0$, and $\mathbf{P}(t)$ consists of $m n-1$ points, counted properly.

Proof: The common intersections of the three curves are given by the roots of

$$
\operatorname{gcd}(f(a, b, c), g(a, b, c))=t^{\alpha-1} s^{\beta} \prod_{i=1}^{m n-\alpha-\beta}\left(\sigma_{i} t-\tau_{i} s\right)
$$

Thus there are $m n-1$ intersections given by the $m n-1$ roots $(t: s)$.

The existence of inversion formulas can be established constructively.

Theorem 3 There exists a degree at most $n-2$ inversion formulat $=f(x, y) / g(x, y)$.

Proof: Let the maximum degree of $f$ and $g$ be $m$. The degree of the numerator of $t g(a(t) / c(t), b(t) / c(t))-$ $f(a(t) / c(t), b(t) / c(t))$ is at most $m n+1$ and its vanishing requires the vanishing of $m n+2$ coefficients that involve $(m+2)(m+1)$ variables (the coefficients of $f$ and $g)$. This is always possible when $(m+2)(m+1)>m n+2$ or $m \geq n-2$.

Intuitively it is clear that the inversion formula has to become the indeterminate $0 / 0$ when $(x, y)$ is a singular point because it corresponds to "more than one" parameter value $t$. The next theorem captures the geometric significance of this observation.

Theorem 4 Algebraically, if $t=f(x, y) / g(x, y)$ and $(x, y)$ is a singular point of $\mathbf{P}$, then $f(x, y) / g(x, y)=0 / 0$. Geometrically, this means the curves $f=0$ and $g=0$ must pass through all the singular points of $\mathbf{P}$.
Proof: If $(x, y)$ is a singular point, then either there are $t_{1} \neq t_{2}$ such that $\mathbf{P}\left(t_{1}\right)=(x, y)=\mathbf{P}\left(t_{2}\right)$; or there is a $t$ such that $(x, y)=\mathbf{P}(t)$ and $a(t) / a^{\prime}(t)=b(t) / b^{\prime}(t)=$ $c(t) / c^{\prime}(t)[5]$.

In the former if $f(x, y) / g(x, y)$ does not evaluate to the indeterminate $0 / 0$ we will have $t_{1}=f(x, y) / g(x, y)=t_{2}$ which is a contradiction.

In the latter we shall proceed in the projective setting. The inversion formula can be re-written as $s f(x, y, w)=$ $\operatorname{tg}(x, y, w)$. Take the partial derivative w.r.t $t$ we have

$s\left(f_{x} x_{t}+f_{x} y_{t}+f_{z} z_{t}\right)=g(x, y, w)+t\left(g_{x} x_{t}+g_{y} y_{t}+g_{z} z_{t}\right)$

At the singularity $(x: y: w)=(a(t): b(t): c(t))$ we may assume without loss of generality that $c(t) \neq 0$. We can then write the above equation as

$s c^{\prime}\left(f_{x} a+f_{x} b+f_{z} c\right)=c g(a, b, c)+t c^{\prime}\left(g_{x} a+g_{y} b+g_{z} c\right)$

Since $f, g$ are homogeneous polynomials of degree $m$, we have $s c^{\prime} m f=c g(a, b, c)+t c^{\prime} m g$ and thus $g(a, b, c)=0$. By taking the partial derivative w.r.t $s$ we similarly conclude that $f(a, b, c)=0$. This completes the claims that the inversion formula evaluates to $0 / 0$ at the singularities of $\mathbf{P}$.

The preceding theorem does not preclude the possibility that $f=0$ and $g=0$ intersect at points other than those of P. An immediate example is linear inversion formulas for lines. The following example is less trivial.

Example. Consider the unit circle (3) and its inversion

$$
t=\frac{-y^{2}+2 w x+2 w^{2}}{y(x+w)} .
$$

The curves $-y^{2}+2 w x+2 w^{2}=0$ and $y(x+w)=0$ intersect three times at $(x: y: w)=(-1: 0: 1)=\mathbf{P}(0)$ and once at $(x: y: w)=(1: 0: 0)$ which is not a point of the unit circle $x^{2}+y^{2}=w^{2}$. Figure 2 shows these intersections on the $x=-1$ plane.

\section{Inversion Formulas of the Lowest Degree}

For $n \geq 1$, inversion formulas from the Sylvester and the Bezout implicitization matrix are in general of degree $n$ and $n-1$ respectively. Theorem 3 shows that actually degree $n-2$ suffices. But lower degree inversion formulas are possible by observing that inversion and parametrization are closely related: inversion solves for $t$ in terms of $x, y$ but parametrization solves for $x, y$ in terms of $t$ [1]. Just like a degree $n$ rational plane curve with a $(n-1)$-ple point can be parametrized using a pencil of lines, a degree $n$ rational plane curve with a $(n-1)$-ple point can be inverted with linear $f$ and $g$. 


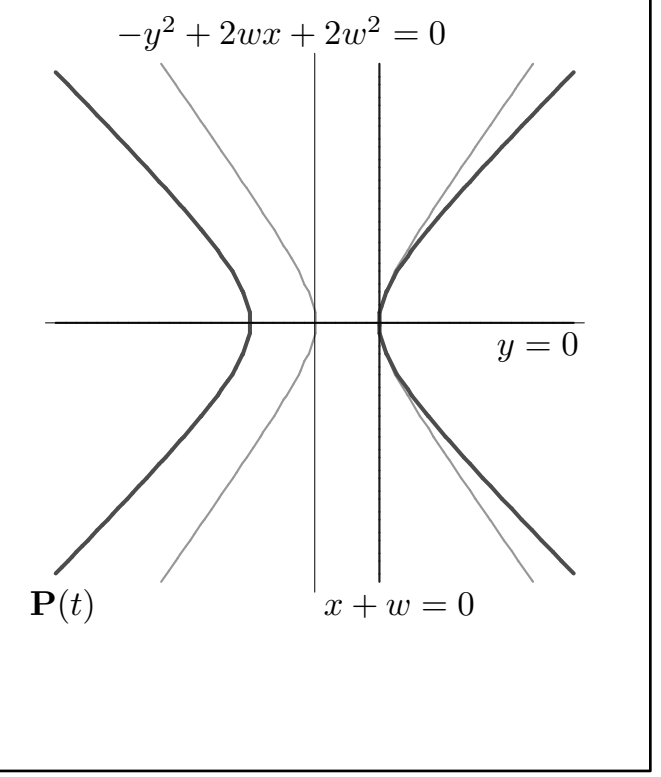

Figure 2. The point $(x: y: w)=(1: 0: 0)$ is not on $\mathbf{P}$ but is an intersection of $-y^{2}+2 w x+2 w^{2}=$ $y(x+w)=0$ on the plane $x=-1$.

\subsection{Lines}

When $n=1$, the Sylvester implicitization matrix

$$
\left[\begin{array}{cc}
c_{1} X-a_{1} & c_{0} X-a_{0} \\
c_{1} Y-b_{1} & c_{0} Y-b_{0}
\end{array}\right]
$$

gives the inversion formulas

$$
t=-\frac{c_{0} Y-b_{0}}{c_{1} Y-b_{1}}, \quad t=-\frac{c_{0} X-a_{0}}{c_{1} X-a_{1}} .
$$

Note that at least one of the inversion formulas will work whether $\mathbf{P}$ is horizontal or vertical. Since $\mathbf{P}(0)=$ $\left(a_{0} / c_{0}, b_{0} / c_{0}\right), \mathbf{P}(\infty)=\left(a_{1} / c_{1}, b_{1} / c_{1}\right)$; clearly the horizontal lines in the first inversion formula pass through $\mathbf{P}(0)$ and $\mathbf{P}(\infty)$ respectively; so are the vertical lines in the second inversion formula.

Other inversion formulas can be created easily. Take any point $(\alpha, \beta, \gamma)$ not on $\mathbf{P}(t)$, it is straightforward to verify that

$$
t=-\frac{\left|\begin{array}{ccc}
x & y & w \\
\alpha & \beta & \gamma \\
a_{0} & b_{0} & c_{0}
\end{array}\right|}{\left|\begin{array}{ccc}
x & y & w \\
\alpha & \beta & \gamma \\
a_{1} & b_{1} & c_{1}
\end{array}\right|}
$$

Example. For the line

$$
\mathbf{P}(t)=\left(\frac{t+4}{2 t+3}, \frac{t-4}{2 t+3}\right)
$$

The Sylvester inversion formulas are

$$
t=-\frac{3 y+4}{2 y-1}, \quad t=-\frac{3 x-4}{2 x-1}
$$

and Formula (26) with $(\alpha: \beta: \gamma)=(0: 0: 1)$, the origin, is

$$
t=\frac{4(x+y)}{x-y} .
$$

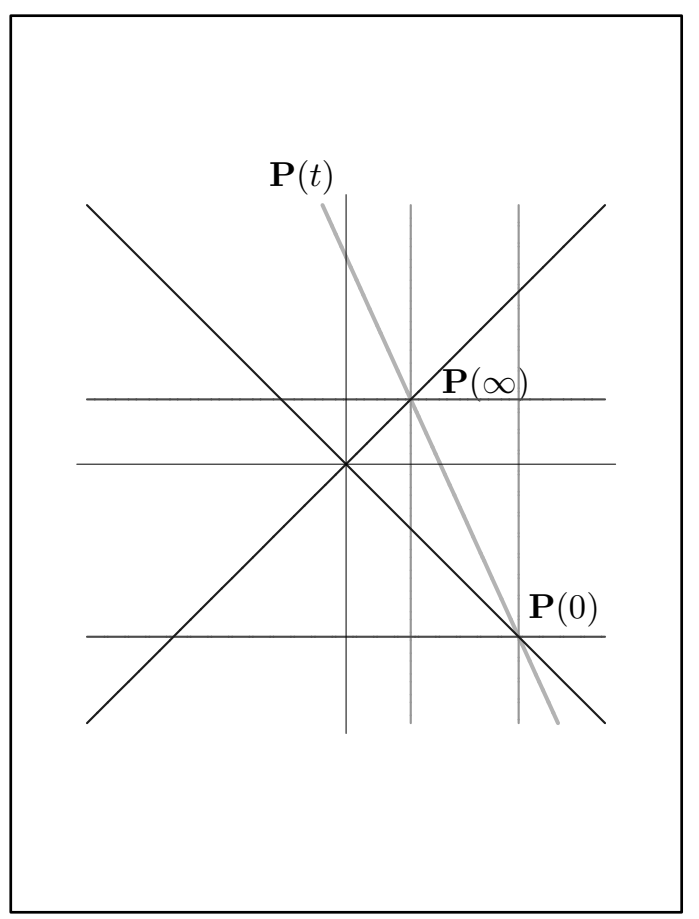

Figure 3. The line $\mathbf{P}(t)=(t+4: t-4: 2 t+3)$ and the inversion lines $3 y+4=1-2 y=0$, $3 x-4=1-2 x=0,4(x+y)=x-y=0$.

\subsection{Conics}

The minor ratios from the Bezout implicitization matrix give linear inversion formulas for conics. They are

$$
t=-\frac{L_{1,0}}{L_{2,0}}, \quad t=-\frac{L_{2,0}}{L_{2,1}},
$$

where

$$
L_{i, j}=\left|\begin{array}{ccc}
x & y & w \\
a_{i} & b_{i} & c_{i} \\
a_{j} & b_{j} & c_{j}
\end{array}\right|
$$


Note that $L_{2,1}$ is tangent to $\mathbf{P}(t)$ at $\mathbf{P}(\infty), L_{2,0}$ intersects $\mathbf{P}(t)$ at $\mathbf{P}(0)$ and $\mathbf{P}(\infty)$, and $L_{1,0}$ is tangent to $\mathbf{P}(t)$ at $\mathbf{P}(0)$.

Again other inversion formulas can be created easily:

$$
t=\frac{1}{t_{0}} \frac{\left|\begin{array}{ccc}
x & y & w \\
x_{0} & y_{0} & w_{0} \\
a_{0} & b_{0} & c_{0}
\end{array}\right|}{\left|\begin{array}{ccc}
x & y & w \\
x_{0} & y_{0} & w_{0} \\
a_{2} & b_{2} & c_{2}
\end{array}\right|}
$$

where $\left(x_{0}: y_{0}: w_{0}\right)=\mathbf{P}\left(t_{0}\right)$ for some parameter $t_{0} \neq$ $0, \infty$.

Example. Using the above formula, inversion formulas for the unit circle (3) for $t=2 / 3$ and $t=-2$ are respectively

$$
t=\frac{3 x-2 y+3}{2 x+3 y-2}, \quad t=\frac{x+2 y+1}{-2 x+y+2}
$$

Figure 4 shows the lines involved in the inversion formulas.

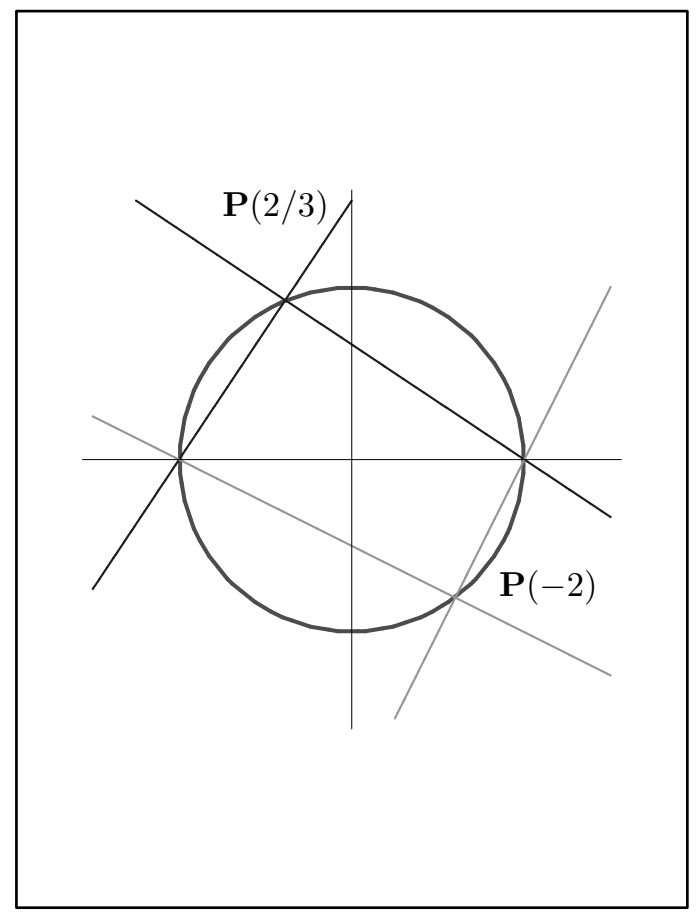

Figure 4. The inversion lines $3 x-2 y+3=$ $2 x+3 y-2=0$ and $x+2 y+1=-2 x+y+2=0$ for the unit circle.

\subsection{Cubics}

Since rational cubics have one double point $D$, a linear inversion formula can be obtained as

$$
t=\frac{L_{0}}{L_{\infty}}
$$

where $L_{0}$ is a line that intersects the cubic at $D$ and $\mathbf{P}(0)$ and $L_{\infty}$ is a line that intersects the cubic at $D$ and $\mathbf{P}(\infty)$. Since each line and the cubic has $1 \times 3=3$ intersections, there are no further intersections by Bezout's theorem.

Example. Clearly the cubic Bezier curve with the control vertices $(0,0),(-1,1),(1,1),(0,0)$

$$
\mathbf{P}(t)=\left(-6 t^{3}+9 t^{2}-3 t,-3 t^{2}+3 t\right)
$$

has a double point $D=(0,0)=\mathbf{P}(0)=\mathbf{P}(1)$ and $\mathbf{P}(\infty)=(1: 0: 0)$. The line passing thru $D$ twice and $\mathbf{P}(\infty)$ once is $y=0$. The line passing thru $D$ three times is $x+y=0$. Indeed we have

$$
t=\frac{x+y}{2 y}
$$

Figure 5 shows the cubic Bezier curve and the lines of inversion.

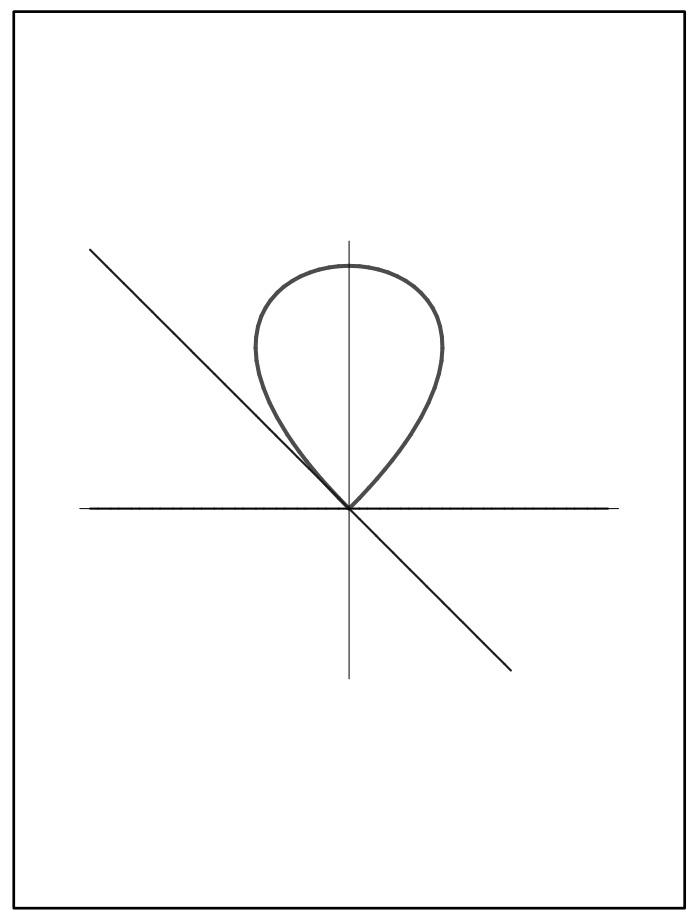

Figure 5. The cubic curve $\mathbf{P}(t)=\left(-6 t^{3}+9 t^{2}-\right.$ $\left.3 t,-3 t^{2}+3 t\right)$ and the inversion lines $x+y=$ $2 y=0$. 


\subsection{Quartics}

A rational quartic has either one triple point $T$ or three distinct double points $D_{1}, D_{2}$, and $D_{3}$. The inversion of a rational quartic is completely determined by its singularity.

Theorem 5 A rational quaritc has a linear inversion if it has a triple point; it has a quadratic but no linear inversion if it has three double points.

Proof: If there is a triple point $T$, a linear inversion formula can be given as $t=L_{0} / L_{\infty}$ where $L_{0}$ is a line intersecting the quartic three times at $T$ and once at $\mathbf{P}(0)$ for a total of four times, $L_{\infty}$ is a line intersecting the quartic three times at $T$ and once at $\mathbf{P}(\infty)$ for a total of also four times.

If there are three distinct double points, the inversion formula $t=f(x, y) / g(x, y)$ cannot be linear. By Theorem 4, bot $f=0$ and $g=0$ have to pass through the three double points. Since the three points are distinct, if $f=0$ and $g=0$ are lines they have to be coincident and their ratio is a constant. However, a quadratic inversion formula can be given as $t=C_{0} / C_{\infty}$ where $\mathbf{P}_{0}$ is a conic intersecting the quartic twice each at the $D_{i}$, once at some simple point $\mathbf{P}\left(t_{0}\right)$, and once at $\mathbf{P}(0)$ for a total of eight intersections; $\mathbf{P}_{\infty}$ is a conic intersecting the quartic similarly except the intersection $\mathbf{P}(0)$ is replaced by $\mathbf{P}(\infty)$.

Example. The quartic curve

$$
\mathbf{P}(t)=\left(\frac{t^{3}-3 t}{\left(1+t^{2}\right)^{2}}, \frac{t^{4}-3 t^{2}}{\left(1+t^{2}\right)^{2}}\right)
$$

has a triple point at the origin: $\mathbf{P}(0)=\mathbf{P}(\sqrt{3})=\mathbf{P}(-\sqrt{3})$. Clearly $t=y / x$ is an inversion formula. The geometric significance is that $y=0$ intersects the quartic four times at $\mathbf{P}(0)$ (the triple point) and $x=0$ intersects the quartic three times at $\mathbf{P}(0)$ and once at $\mathbf{P}(\infty)$. Figure 6 shows the quartic and the lines for the inversion. Note that we cannot take the lines $\sqrt{3} x \pm y=0$ though they each intersects the quartic four times at the origin because the extra intersection has to correspond to $t=0$.

Example. Consider a rational Bezier quartic

$$
\begin{aligned}
\mathbf{P}(t)= & \left(\frac{52 t^{3}-78 t^{2}+28 t-1}{16 t^{4}-32 t^{3}+12 t^{2}+4 t+1},\right. \\
& \left.\frac{-70 t^{4}+140 t^{3}-90 t^{2}+20 t}{16 t^{4}-32 t^{3}+12 t^{2}+4 t+1}\right)
\end{aligned}
$$

whose control vertices $(x: y: w)$ are $(-1: 0: 1),(6$ : $5: 2),(0:-5: 5),(-6: 5: 2),(1: 0: 1)$. A conic that passes through $\mathbf{P}(1 / 2), \mathbf{P}(0)$, and the three double points is

$$
\begin{aligned}
& 130975 x^{2}-859240 x y+1155792 y^{2} \\
& +267100 x-894190 y+136125=0
\end{aligned} .
$$

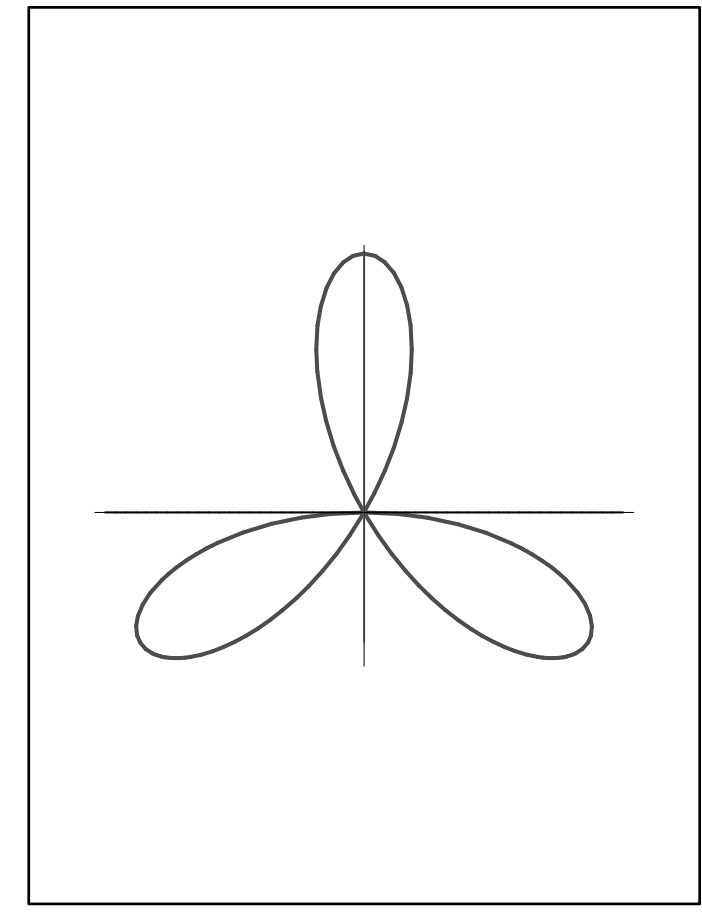

Figure 6. The quartic $\mathbf{P}(t)=\left(\frac{t^{3}-3 t}{\left(1+t^{2}\right)^{2}}, \frac{t^{4}-3 t^{2}}{\left(1+t^{2}\right)^{2}}\right)$ and the inversion lines $y=x=0$.

A conic that passes through $\mathbf{P}(1 / 2), \mathbf{P}(\infty)$, and the three double points is

$$
-40 x(42962 y-13355)=0 .
$$

Figure 7 shows the quartic with its three double points and the inversion conics.

\subsection{Higher Degree Rational Curves}

To invert higher degree rational plane curves, we can either use the ratios of the first minors of the implicitization Bezout matrix to obtain degree at most $n-1$ inversion formulas or solve a system of linear equations by equating the coefficients of powers of $t$ to zero in

$$
\operatorname{tg}(a(t), b(t), c(t))-f(a(t), b(t), c(t)) \equiv 0
$$

(here $g, f$ are the homogeneous version) to find degree at most $n-2$ inversion formulas.

To determine the lowest degree inversion formulas, a brute-force approach is to try to solve Equation (41) with $m$ from 1 to $n-2$. It should be noted that the lowest degree is determined by the singularity of $\mathbf{P}$. For example, a rational quintic with a quadruple point has a linear inversion. A rational quintic with six double points in general positions has a cubic inversion which is the lowest degree inversion. This is so because a line or a conic cannot go through six points in general positions. 


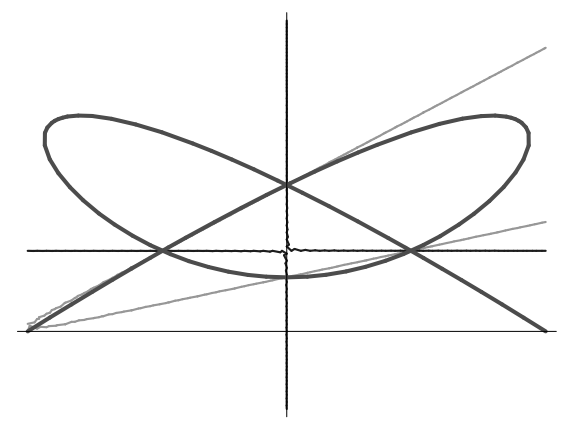

Figure 7. A quartic with three double points and the inversion conics.

\section{Inversion of Fat Curves}

It turns out that if $(x, y)$ is a point near $\mathbf{P}$ then an inversion formula can be obtained that finds a curve point $\mathbf{P}(t)$ that is nearest $(x, y)$. Observe that if $t=f(x, y) / g(x, y)$, then the moving curve $V(x, y ; t) \equiv f(x, y)-t g(x, y)=0$ intersects $\mathbf{P}$ at $\mathbf{P}(t)$. If $V=0$ intersects $\mathbf{P}$ orthogonally at $\mathbf{P}(t)=\left(x_{1}, y_{1}\right)$, then for any $\left(x_{2}, y_{2}\right)$ on $V=0$, we have $f\left(x_{2}, y_{2}\right) / g\left(x_{2}, y_{2}\right)=f\left(x_{1}, y_{1}\right) / g\left(x_{1}, y_{1}\right)=t$ and $\left(x_{1}, y_{1}\right)$ is the curve point nearest $\left(x_{2}, y_{2}\right)$. We may consider $\mathbf{P}$ together with points near it as forming a fat curve. The above argument shows that if the curve $f-t g=0$ intersects $\mathbf{P}$ orthogonally, then the inversion formula $t=f / g$ inverts points on the fat curve by finding the parameter of the curve point nearest a given point.

Let the degrees of $f$ and $g$ be $m$. The conditions needed for orthogonal intersection are

$$
f\left(\frac{a(t)}{c(t)}, \frac{b(t)}{c(t)}\right)-t g\left(\frac{a(t)}{c(t)}, \frac{b(t)}{c(t)}\right) \equiv 0
$$

and

$$
\frac{\left(f_{x}-t g_{x}\right)\left(\frac{a(t)}{c(t)}, \frac{b(t)}{c(t)}\right)}{\frac{a^{\prime}(t) c(t)-a(t) c^{\prime}(t)}{c(t)^{2}}} \equiv \frac{\left(f_{y}-t g_{y}\right)\left(\frac{a(t)}{c(t)}, \frac{b(t)}{c(t)}\right)}{\frac{b^{\prime}(t) c(t)-b(t) c^{\prime}(t)}{c(t)^{2}}}
$$

The first identity imposes $m n+2$ conditions and the second identity imposes $m n+n$ conditions. The polynomials $f$ and $g$ supply $(m+2)(m+1)$ coefficients as variables. Algebraically it is always possible to find $m$ such that

$$
m^{2}+3 m>2 m n+n
$$

(the condition needed is $m^{2}+3 m>2 m n+1$ when $\mathbf{P}$ is polynomial). But when $m \geq n$ a solution is usable for inversion only when $f$ and $g$ are not multiples of the implicit equation of $\mathbf{P}$.

Example. An inversion formula for a fat line is

$$
t=-\frac{c_{0} A x+c_{0} B y-C_{1} w}{c_{1} A x+c_{1} B y-C_{2} w}
$$

where

$$
\begin{gathered}
A=\left|\begin{array}{cc}
a_{1} & a_{0} \\
c_{1} & c_{0}
\end{array}\right|, B=\left|\begin{array}{cc}
b_{1} & b_{0} \\
c_{1} & c_{0}
\end{array}\right|, \\
C_{1}=\left|\begin{array}{cc}
a_{0} a_{1}+b_{0} b_{1} & a_{0}^{2}+b_{0}^{2} \\
c_{1} & c_{0}
\end{array}\right|, \\
C_{2}=\left|\begin{array}{cc}
a_{1}^{2}+b_{1}^{2} & a_{0} a_{1}+b_{0} b_{1} \\
c_{1} & c_{0}
\end{array}\right| .
\end{gathered}
$$

It is easy to see that $t\left(c_{1} A x+c_{1} B y-C_{2} w\right)+\left(c_{0} A x+\right.$ $\left.c_{0} B y-C_{1} w\right)=0$ indeed is a line orthogonal to $\mathbf{P}$ at $\mathbf{P}(t)$.

Example. Inversions formulas for a fat unit circle centered at the origin include

$$
t=\frac{(x+1)^{2}+y^{2}}{2 y}, \quad t=\frac{2 y}{(x-1)^{2}+y^{2}} .
$$

The circle $2 t y-\left((x+1)^{2}+y^{2}\right)=0$ is centered at $(-1, t)$ with radius $|t|$. The circle $t\left((x-1)^{2}+y^{2}\right)-2 y=0$ is centered at $(1,1 / t)$ with radius $1 /|t|$. Figures 8 illustrates these orthogonal circles.

\section{Flatter Inversion Curves}

The inversion curve $V(x, y ; t) \equiv t g(x, y)-f(x, y)=$ 0 can be made not only orthogonal but also flatter. Let a normal to $\mathbf{P}(t)$ be

$$
N(t)=\left(n_{x}(t), n_{y}(t)\right)=\mathbf{P}^{\prime}(t)\left[\begin{array}{cc}
0 & -1 \\
1 & 0
\end{array}\right] .
$$

In general the degrees of the numerators of $N(t)$ are $2 n-2$. Consider the intersection of $V(x, y ; t)=0$ and the normal $\mathbf{P}(t)+\lambda N(t):$

$$
V(\mathbf{P}(t)+\lambda N(t))=\sum_{k=0}^{m} \frac{\lambda^{k}}{k !} \sum_{i=0}^{k}\left(\begin{array}{c}
k \\
i
\end{array}\right) \frac{\partial^{k} V(\mathbf{P}(t))}{\partial x^{k-i} \partial y^{i}} n_{x}^{k-i} n_{y}^{i}
$$




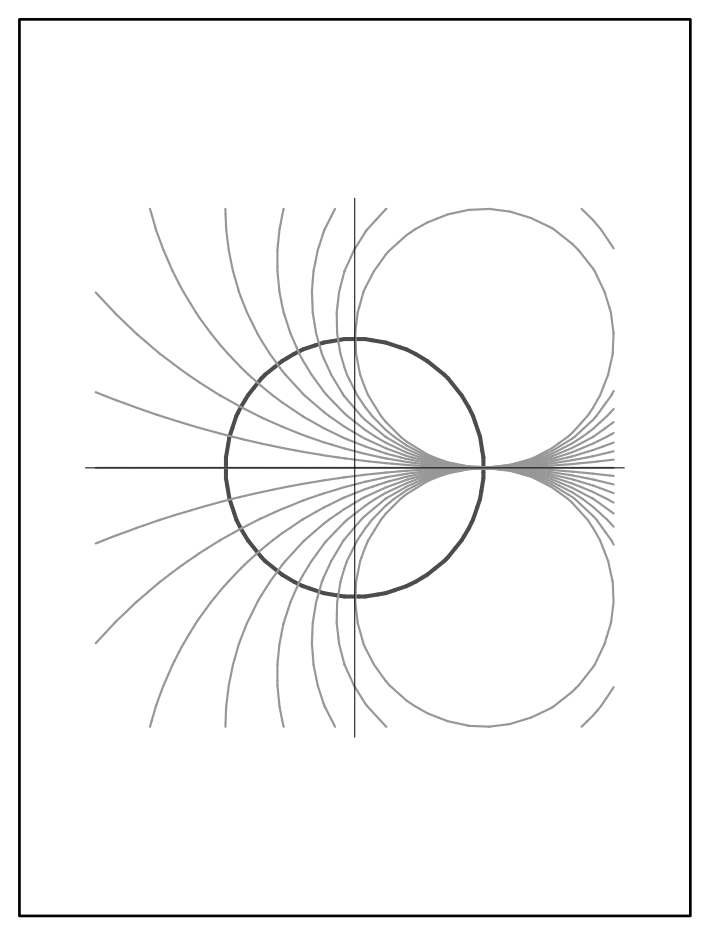

Figure 8. The curves $t\left((x-1)^{2}+y^{2}\right)-2 y=0$ for $t=0.125 N, N=-8,-7, \cdots, 8$.

The degree of the numerator of $\frac{\partial^{k} V(\mathbf{P}(t))}{\partial x^{k-i} \partial y^{i}} n_{x}^{k-i} n_{y}^{i}$ in $t$ is $m n+k n+1-2 k$. Thus if $V=0$ and the normal is to have an intersection of multiplicity $\mu$ at $\mathbf{P}(t)$, the number of constraints to be satisfied is

$\sum_{k=0}^{\mu-1}(m n+k n+2-2 k)=\mu\left(m n+\frac{(\mu-1)(n-2)}{2}+2\right)$

which is $O(m)$. Since $f$ and $g$ altogether supplies $(m+$ $2)(m+1)$ variables, which is $O\left(m^{2}\right)$, the constraints can always be satisfied algebraically. Just like fat curve inversion, when $m \geq n$, a solution $f, g$ is usable for inversion only when $f, g$ are not multiples of the implicit equation of P.

The following inversion curves and the normals of the unit circle centered at the origin have triple intersections:

$$
\begin{aligned}
& t=\frac{3 x^{2}+3 y^{2}+1+x\left(x^{2}+y^{2}+3\right)}{y\left(x^{2}+y^{2}+3\right)} \\
& t=\frac{y\left(x^{2}+y^{2}+3\right)}{3 x^{2}+3 y^{2}+1-x\left(x^{2}+y^{2}+3\right)}
\end{aligned}
$$

These inversion curves are shown in Figures 9, 10.

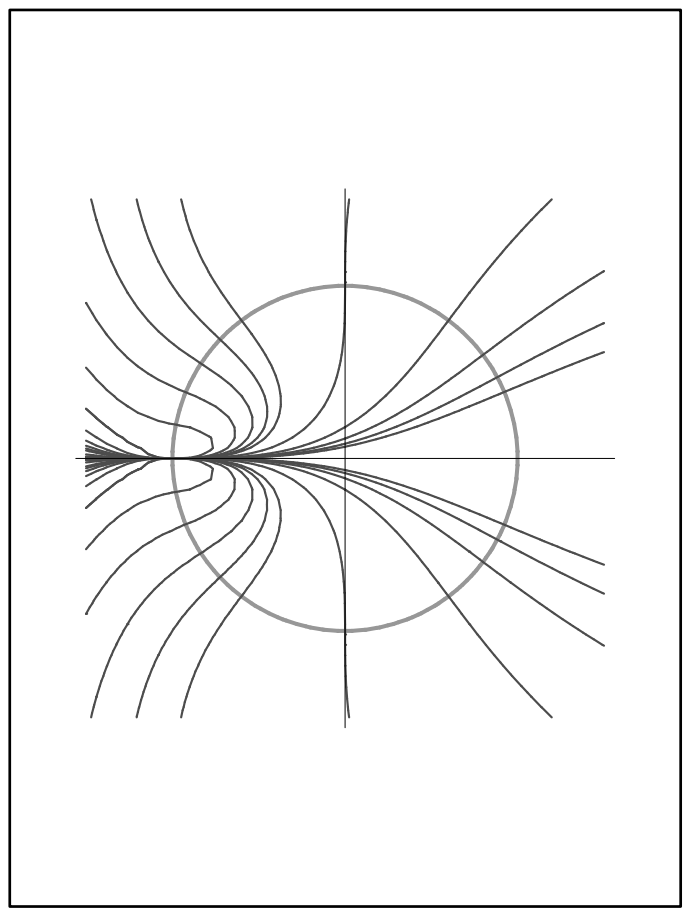

Figure 9. The inversion curves $t y\left(x^{2}+y^{2}+3\right)-$ $3\left(x^{2}+y^{2}\right)-1-x\left(x^{2}+y^{2}+3\right)$ for $t=0.1 N, N$; $N=-5,-4, \cdots, 5$.

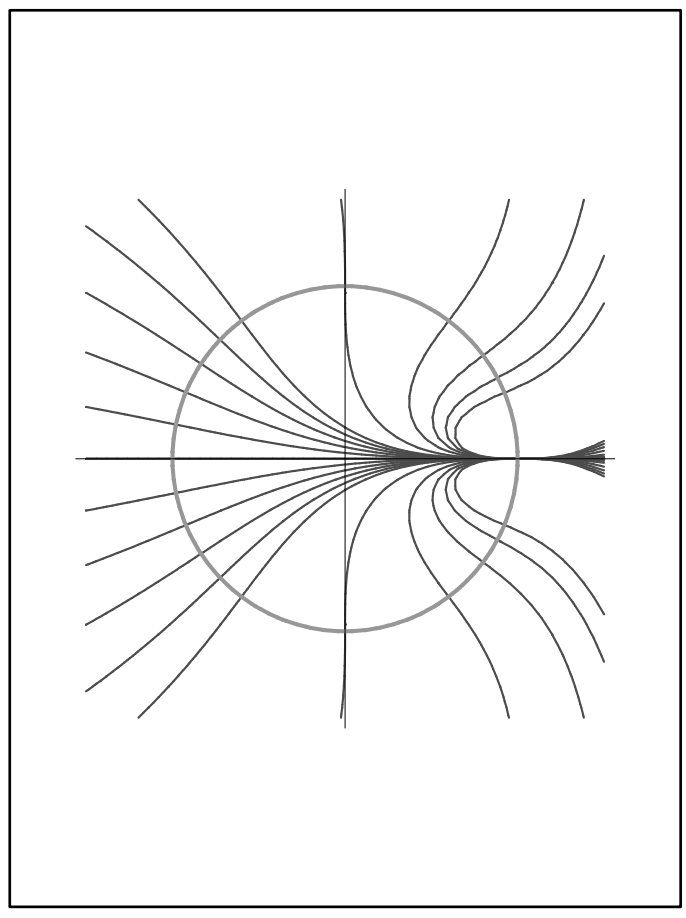

Figure 10. The inversion curves $t\left(3\left(x^{2}+y^{2}\right)+\right.$ $\left.1-x\left(x^{2}+y^{2}+3\right)\right)-y\left(x^{2}+y^{2}+3\right)$ for $t=0.1 N, N$; $N=-5,-4, \cdots, 5$. 


\section{Space Curve Inversion}

Consider a degree $n$ space curve whose points $(x: y$ : $z: w)$ are given by

$$
\mathbf{P}(t)=(a(t): b(t): c(t): d(t))
$$

where $(a(t): b(t): c(t): d(t))=\sum_{i=0}^{n}\left(a_{i}: b_{i}: c_{i}: d_{i}\right) t^{i}$ are lowest form polynomials and not all of $a_{n}, b_{n}, c_{n}, d_{n}$ are zero. If we simply take some projection of the space curve on a plane we will have a plane curve which may or may not be properly parametrized. However, an inversion formula of much lower degree can always be obtained algebraically as shown below.

Let $f(x, y, z), g(x, y, z)$ be degree $m$ polynomials such that

$$
f\left(\frac{a}{d}, \frac{b}{d}, \frac{c}{d}\right)-\operatorname{tg}\left(\frac{a}{d}, \frac{b}{d}, \frac{c}{d}\right) \equiv 0 .
$$

This requires the solving of $m n+2$ linear equations in $2\left(\begin{array}{c}m+3 \\ 3\end{array}\right)$ variables. Thus if

$$
\frac{(m+3)(m+2)(m+1)}{3}>m n+2
$$

there is a solution. Simple computation gives the smallest solution $m$ for $1 \leq n \leq 12$ :

\begin{tabular}{|c|c|}
\hline$n$ & $m$ \\
\hline $1 . .5$ & 1 \\
$6 . .8$ & 2 \\
$9 . .12$ & 3 \\
\hline
\end{tabular}

Example. Consider the space curve

$$
\mathbf{P}(t)=\left(t^{4}-1: 2 t^{2}: t^{3}\left(t^{4}+1\right): t^{4}+1\right) .
$$

If we take the projection from $(x: y: z: w)=(0: 0: 1$ : 0 ) on the plane $z=0$ the projected plane curve is

$$
C(t)=\left(\frac{t^{4}-1}{t^{4}+1}, \frac{2 t^{2}}{t^{4}+1}\right)
$$

which is clearly improperly parametrized since $C(t)=$ $C(-t)$.

But a degree 2 inversion formula can be found by solving Equation (56):

$$
t=\frac{\left(l_{1}+l_{4}\right) y z+l_{2}(x+1)+l_{3}(1-x) z}{l_{1}(x+1)+l_{2}(1-x) z+l_{3} y+l_{4}\left(x^{2}+y^{2}+x\right)}
$$

where $l_{i}$ 's are arbitrary constants. Simpler degree 2 inversion formulas derived from the above include

$$
\begin{aligned}
& t=\frac{x+1}{z(1-x)}, \quad t=\frac{z(1-x)}{y}, \\
& t=\frac{y z}{x+1}, \quad t=\frac{y z}{x^{2}+y^{2}+x} .
\end{aligned}
$$

\section{Surface Inversion}

A total degree $n$ rational surface may be given as

$$
\mathbf{P}(t, u)=(a(t, u): b(t, u): c(t, u): d(t, u))
$$

where $(a: b: c: d)=\sum_{i+j \leq n}\left(a_{i, j}, b_{i, j}, c_{i, j}, d_{i, j}\right) s^{i} t^{j}$ are lowest form polynomials and at least one of $a, b, c, d$ is of degree $n$. Such surfaces arise when triangular patches are used.

Algebraically, an inversion formula for $t$ can be found by solving

$$
f\left(\frac{a}{d}, \frac{b}{d}, \frac{c}{d}\right)-\operatorname{tg}\left(\frac{a}{d}, \frac{b}{d}, \frac{c}{d}\right) \equiv 0
$$

where $f(x, y, z)$ and $g(x, y, z)$ are degree $m>0$ polynomials. Since $f$ and $g$ provide $2\left(\begin{array}{c}m+3 \\ 3\end{array}\right)$ coefficients and the numerator after the substitution is a degree $m n+1$ polynomials in two variables without the $u^{m n+1}$ term, the inequality

$$
2\left(\begin{array}{c}
m+3 \\
3
\end{array}\right)>\left(\begin{array}{c}
m n+3 \\
2
\end{array}\right)-1
$$

ensures a solution. The condition for an inversion formula for $u$ can be obtained similarly. Since $m$ becomes very large quickly Inequality (66) does not seem to produce a useful bound for the inversion formula.

In CAGD, rectangular, or tensor product, patches are very common. Algebraically a rectangular patch is a bidegree $\left(n_{1}, n_{2}\right)$ surface whose total degree is $n_{1}+n_{2}$. It is defined by Equation (64) but here the polynomials $a, b, c, d$ are of degree $n_{1}$ in $t$ and $n_{2}$ in $u$.

Since bi-degree $\left(n_{1}, n_{2}\right)$ surfaces can be implicitized by the Dixon resultant [6], Rectangular patches can also be inverted by ratios of first minors of the implicitization Dixon matrix. In general the implicitization Dixon matrix has order $2 n_{1} n_{2}$, thus the inversion formulas obtained as ratios of Dixon matrix first minors has degree $2 n_{1} n_{2}-1$.

It is known that the Dixon method survives rectangular corner cutting [2], consequently the method of minors for inversion applies when the rectangular bi-degree monomial support undergoes rectangular corner cutting. If the number of monomials removed is $r$, then the degree of the inversion formula is $2 n_{1} n_{2}-1-r$ because the Dixon matrix here has order $2 n_{1} n_{2}-r$.

Example. Consider the monomial configuration:

$$
\begin{array}{c|cccc}
u^{3} & \circ & \circ & \bullet & \circ \\
u^{2} & \circ & \circ & \bullet & \circ \\
u & \bullet & \bullet & \bullet & \circ \\
1 & \circ & \circ & \circ & \bullet \\
\hline & 1 & t & t^{2} & t^{3}
\end{array}
$$

That is, the polynomials $a(t, u), b(t, u), c(t, u), d(t, u)$ involves only only monomials $u, t u, t^{2} u, t^{2} u^{2}, t^{2} u^{3}, t^{3}$. 
For this monomial support the Dixon matrix is of order $2 \times 3 \times 3-10=8$ and the inversion formulas from the ratios of its minors are of degree at most 7 .

\section{Conclusion}

The paper discussed three methods of finding an inversion formula for curves and surfaces. The methods are the implicitization matrx first minor ratios, the ratio of implicit curves that intersect the curve in a certain manner, and linear algebra. Important properties of inversion formulas include their degrees and their ability to invert points that are near the curve. The interplay between curve singularity and lowest degree inversion formula was also explored. The paper also found that space curves have relatively lower degree inversion formulas. The discussion on surface inversion was quite preliminary. Much remains to be done concerning total degree surface inversion. In particular, the relationship between surface singularity and inversion degree deserves further research.

\section{References}

[1] S. S. Abhyankar. Algebraic geometry for scientists and engineers. American Mathematical Society, Providence, R.I., 1990.

[2] E. W. Chionh. Rectangular corner cutting and dixon $\dashv-$ resultants. Journal of Symbolic Computation, 31:651-669, 2001.

[3] E. W. Chionh and T. W. Sederberg. On the minors of the implicitization Bezout matrix for a rational plane curve. Computer Aided Geometric Design, 18(1):21-36, 2001.

[4] R. Goldman, T. Sederberg, and D. Anderson. Vector elimination: A technique for the implicitization, inversion and intersection of planar parametric rational polynomial curves. Computer Aided Geometric Design, 1:327-356, 1984.

[5] H. Hilton. Plane Algebraic Curves. Clarendon Press, Oxford, 1920.

[6] T. Sederberg, D. Anderson, and R. Goldman. Implicit representation of parametric curves and surfaces. Computer Vision, Graphics and Image Processing, 28:72-84, 1984.

[7] R. J. Walker. Algebraic Curves. Dover Publications, Inc., New York, 1950.

[8] W. Wang and J. Barry. The geometric interpretation of inversion formulae for rational plane curves. Computer Aided Geometric Design, 12(5):469-489, 1995. 